\title{
MiR-146a regulates regulatory $T$ cells to suppress heart transplant rejection in mice
}

\author{
Jian Lu (iD) ${ }^{1,4}$, Weiwei Wang (D) ${ }^{1,2,4}$, Peiyuan Li (D) ${ }^{1,4}$, Xiaodong Wang (D) ${ }^{3}$, Chao Gao (D) ${ }^{1}$, Baotong Zhang (iD ${ }^{1}$, Xuezhi Du (D) ${ }^{1}$, \\ Yanhong Liu (iD) ${ }^{1}$, Yong Yang (D) $^{1}$ and Feng Qi ${ }^{1{ }^{1}}$
}

(c) The Author(s) 2021

Regulatory T cells (Tregs), which characteristically express forkhead box protein 3 (Foxp3), are essential for the induction of immune tolerance. Here, we investigated microRNA-146a (miR-146a), a miRNA that is widely expressed in Tregs and closely related to their homeostasis and function, with the aim of enhancing the function of Tregs by regulating miR-146a and then suppressing transplant rejection. The effect of the absence of miR-146a on Treg function in the presence or absence of rapamycin was detected in both a mouse heart transplantation model and cell co-cultures in vitro. The absence of miR-146a exerted a mild tissue-protective effect by transiently prolonging allograft survival and reducing the infiltration of $\mathrm{CD}^{+}$and $\mathrm{CD} 8^{+} \mathrm{T}$ cells into the allografts. Meanwhile, the absence of miR-146a increased Treg expansion but impaired the ability of Tregs to restrict T helper cell type 1 (Th1) responses. A miR-146a deficiency combined with interferon (IFN)- $\gamma$ blockade repaired the impaired Treg function, further prolonged allograft survival, and alleviated rejection. Importantly, miR-146a regulated Tregs mainly through the IFN- $\gamma /$ signal transducer and activator of transcription (STAT) 1 pathway, which is implicated in Treg function to inhibit Th1 responses. Our data suggest miR-146a controls a specific aspect of Treg function, and modulation of miR-146a may enhance Treg efficacy in alleviating heart transplant rejection in mice.

Cell Death Discovery (2021)7:165; https://doi.org/10.1038/s41420-021-00534-9

\section{INTRODUCTION}

Organ transplantation is an effective treatment for patients with end-stage organ failure, but immune rejection after transplantation is still one of the main factors affecting the survival rate and quality of life of patients [1-3]. Although the application of various immunosuppressants may provide temporary symptom relief, unfortunately, these drugs have significant side effects [3-5].

Regulatory $\mathrm{T}$ cells (Tregs), which are characterized by the expression of the transcription factor forkhead box protein 3 (Foxp3), are a special population with immunoregulatory functions that inhibits the excessive activation of self-antigens, monitors the expansion of lymphocytes, and effectively suppresses excessive immune responses [6-8]. Adoptive transfer of Tregs effectively alleviates islet autoimmunity in mice [9]. Clinical trials have shown that the in vitro expansion and reinfusion of Tregs reduces the incidence of acute graft-versus-host disease and prolongs patient survival [10]. Therefore, increasing the number of Tregs or strengthening their immunoregulatory function may ameliorate rejection and induce immune tolerance. While a sufficient number of Tregs is induced in vitro at the early stage of clinical transplantation, several factors, such as low purity, insufficient function, and variable phenotype, limit the infusion of Tregs, and the adoptive transfer of Tregs alone is not sufficient to induce immune tolerance, suggesting that a different therapeutic approach is required to overcome these shortcomings [11-14].

MicroRNAs (miRNAs) are endogenous, noncoding, singlestranded RNA molecules with a length of approximately 22 nucleotides that are fully complementary to the $3^{\prime}$-UTR of their target $m R N A$, leading to $m R N A$ cleavage or translational inhibition $[15,16]$. MiRNAs play an important regulatory role in the homeostasis and function of Tregs in both normal and inflammatory environments [17-19]. Notably, miR-146a, which is expressed at high levels in Tregs relative to conventional $\mathrm{CD}^{+}{ }^{+} \mathrm{T}$ cells [20], negatively regulates the activation of the NF-KB pathway by directly targeting TRAF6 and IRAK1 to control the inflammatory response $[21,22]$. Other studies have shown that the expression and activation of signal transducer and activator of transcription (STAT) 1, one of the targets of miR-146a, is critical for Tregs to control interferon (IFN)- $\gamma$-mediated $T$ helper cell type 1 (Th1) immune responses and associated inflammation [23]. However, the function and molecular mechanism of miR-146a expressed in Tregs in organ transplantation remain unclear.

This study found that the absence of miR-146a in Tregs promoted Treg survival but impaired their function. A miR-146a deficiency exerted a synergistic effect with IFN- $\gamma$ neutralization on enhancing Treg function and alleviating rejection. Mechanistically,

\footnotetext{
${ }^{1}$ Department of General Surgery, Tianjin Medical University General Hospital, Anshan Road, Tianjin 300052, China. ${ }^{2}$ Department of General Surgery, Tianjin Medical University Baodi Clinical College, Guangchuan Road, Tianjin 301800, China. ${ }^{3}$ Department of Gastrointestinal Surgery, The First Affiliated Hospital, School of Medicine, Zhejiang University, Qingchun Road, Hangzhou 310003 Zhejiang Province, China. ${ }^{4}$ These authors contributed equally: Jian Lu, Weiwei Wang, Peiyuan Li. Edited by Ivano Amelio. 凶email: qf@medmail.com.cn
} 
miR-146a regulated Tregs mainly through the IFN- - /STAT1 pathway during rejection.

\section{RESULTS}

\section{Increased miR-146a expression in Tregs during rejection}

We compared the expression of miR-146a in Tregs between the isogeneic transplant group (WT $\rightarrow$ WT) and the allogeneic transplant group (BALB/c $\rightarrow$ WT) to identify the regulatory effect of miR-146a on rejection. The results showed increased miR-146a expression in the Tregs of allogeneic transplant recipients on the $3 \mathrm{rd}$, 5th, and 7th days after transplantation in a mouse heart transplantation model compared with those of the isogeneic transplant recipients (Fig. 1). Therefore, miR-146a is involved in the immune rejection process, and altering miR-146a expression may suppress rejection.

\section{The absence of miR-146a slightly inhibited rejection after transplantation}

According to a previous report, miR-146a ${ }^{-1-}$ mice develop severe lymphatic and myelodysplastic syndromes at 6 months of age [23]; however, miR-146a CKO mice aged 6-8 weeks were used in our study, and no clinical signs of autoimmunity and inflammation were observed.

After the generation of CKO mice, we first analyzed the survival time of the allografts to determine the effect of the absence of miR-146a on rejection in the mouse heart transplantation model. As shown in Fig. 2A, the median survival time (MST) in the CKO group was transiently prolonged from 7 days to 11 days (MST in the WT group, 7 days; MST in the CKO group, 11 days; $p<0.01$ ), and the MST in the CKO+RAPA combination group was further prolonged to 20.5 days (MST in the CKO group, 11 days; MST in the CKO+RAPA group, 20.5 days; $p<0.001)$. The histological changes in the allografts on the 5 th day after transplantation showed a low level of inflammatory cell infiltration, well-preserved myocardial structure, and mild vasculopathy in the CKO group compared with the WT group (Fig. 2C). Moreover, significant differences were observed in the PR scoring and CAV grading of H\&E staining, and these changes were further alleviated in the CKO+RAPA group (Fig. 2B). In addition, Masson's staining of the allografts on the 5th day after transplantation showed that the fibrotic area of allografts in the CKO group was reduced compared with the WT group, while the fibrotic area of allografts in the CKO+RAPA group was even further reduced compared with that of the CKO group (Fig. 2D). Based on these results, the absence of miR-146a transiently prolonged allograft survival time and reduced histological damage, and a synergistic effect was observed in combination with RAPA treatment.

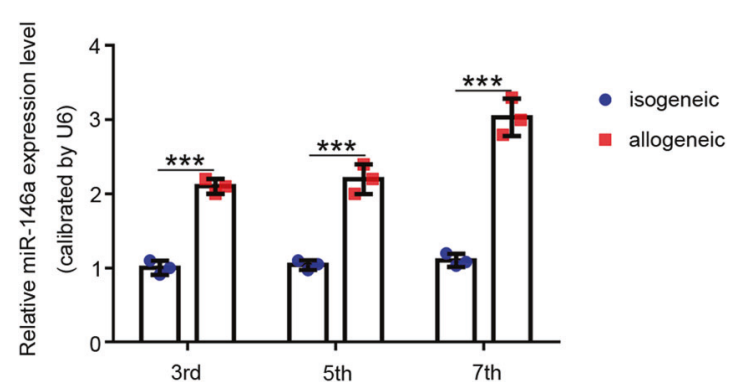

Fig. 1 Differences in the expression levels of miR-146a in isogeneic and allogeneic transplants. The expression level of miR-146a in Tregs isolated from the spleens of isogeneic (WT $\rightarrow$ WT) and allogeneic transplant (BALB/c $\rightarrow$ WT) recipients on days 3,5 , and 7 after transplantation, $n=3$. Data are presented as means \pm SEM. $* * * p<0.001$.
The different degrees of infiltration by $T$ lymphocytes and Tregs in the allografts are closely related to tissue damage. On the 5th day after transplantation, the proportions of infiltrated $\mathrm{CD}^{+}$and $\mathrm{CD}^{+} \mathrm{T}$ cells detected in the allografts using immunofluorescence staining were decreased in the CKO group (Fig. 2E), while the proportion of infiltrated Tregs (Foxp $3^{+}$) detected using immunohistochemistry was increased (Fig. 2F) compared with the WT group. In addition, the combination of CKO+RAPA further reduced the proportion of $\mathrm{CD}^{+}{ }^{+} \mathrm{T}$ cells compared with the CKO group (Fig. 2E).

High mobility group box 1 (HMGB1) [24] and Troponin T [25] are important mediators of myocardial inflammation and heart failure. On the 5th day after transplantation, both HMGB1 expressions (Fig. 3A) detected in allografts using immunohistochemistry and serum Troponin $\mathrm{T}$ levels (Fig. 3B) detected using ELISAs were decreased in recipients in the CKO group compared with the WT group, and HMGB1 and Troponin T levels were both decreased in the CKO + RAPA group compared with the CKO group (Fig. 3A-B).

Thus, the absence of miR-146a slightly alleviates rejection, with synergistic therapeutic effects observed in combination with RAPA treatment.

\section{Effect of the absence of miR-146a on the immune response of transplant recipients}

Flow cytometry was used to analyze each cell type in the recipient's spleens on the 5th day after transplantation and to investigate the effect of miR-146a on the immune response in the recipients. As shown in Fig. $3 \mathrm{C}$, the proportions and numbers of Tregs $\left(\mathrm{CD}^{+}{ }^{+} \mathrm{Foxp}^{+}\right)$were increased in the CKO group compared with those in the WT group. The proportions and numbers of Tregs were further increased in the CKO+RAPA group compared with those in the CKO group.

Macrophage polarization was also assessed in the recipients. As shown in Fig. 3D, compared with those in the WT group, the proportions and numbers of $\mathrm{M} 1$ macrophages $\left(\mathrm{CD} 16 / 32^{+} \mathrm{CD} 68^{+}\right.$) were decreased in the CKO group, while the proportions and numbers of $\mathrm{M} 2$ macrophages $\left(\mathrm{CD} 206^{+} \mathrm{CD}^{+} 8^{+}\right)$increased. The proportions and numbers of M1 macrophages in the CKO+RAPA group were further decreased, while the proportions and numbers of $\mathrm{M} 2$ macrophages were further increased compared with those in the CKO group.

In addition, the expression levels of inflammatory factors in the serum of recipient mice were detected using ELISAs. Compared with the WT group, the levels of the anti-inflammatory factors IL10 and TGF- $\beta 1$ were increased in the CKO group (Fig. 3E-F). CKO combined with RAPA further increased IL-10 and TGF- $\beta 1$ levels compared with those in the CKO group (Fig. 3E-F).

These results suggest that the absence of miR-146a in Tregs not only increases the number of Tregs in transplant recipients but also promotes M2 macrophage polarization.

\section{The absence of miR-146a promoted the expansion of Tregs in vivo}

We examined the proportions and numbers of Tregs in the peripheral blood, spleen, and thymus of the WT and CKO mice using flow cytometry to evaluate the effect of miR-146a on Treg proliferation. The results showed increased proportions (Fig. 4A-B) and numbers (Fig. 4C-D) of Tregs in the peripheral blood and spleen, but not in the thymus, of the CKO group compared with the WT group. Consistent with their increased proliferation, CKO Tregs expressed high levels of Ki67 (Fig. 4E).

Furthermore, we tested the expression levels of inflammatory cytokines in serum. The ELISA results showed increased levels of the anti-inflammatory cytokines IL-10 and TGF- $\beta 1$ in the CKO group compared with their levels in the WT group (Fig. 4F-G).

These results are consistent with those obtained from transplant recipients, suggesting that the absence of miR-146a promotes Treg expansion in vivo. 

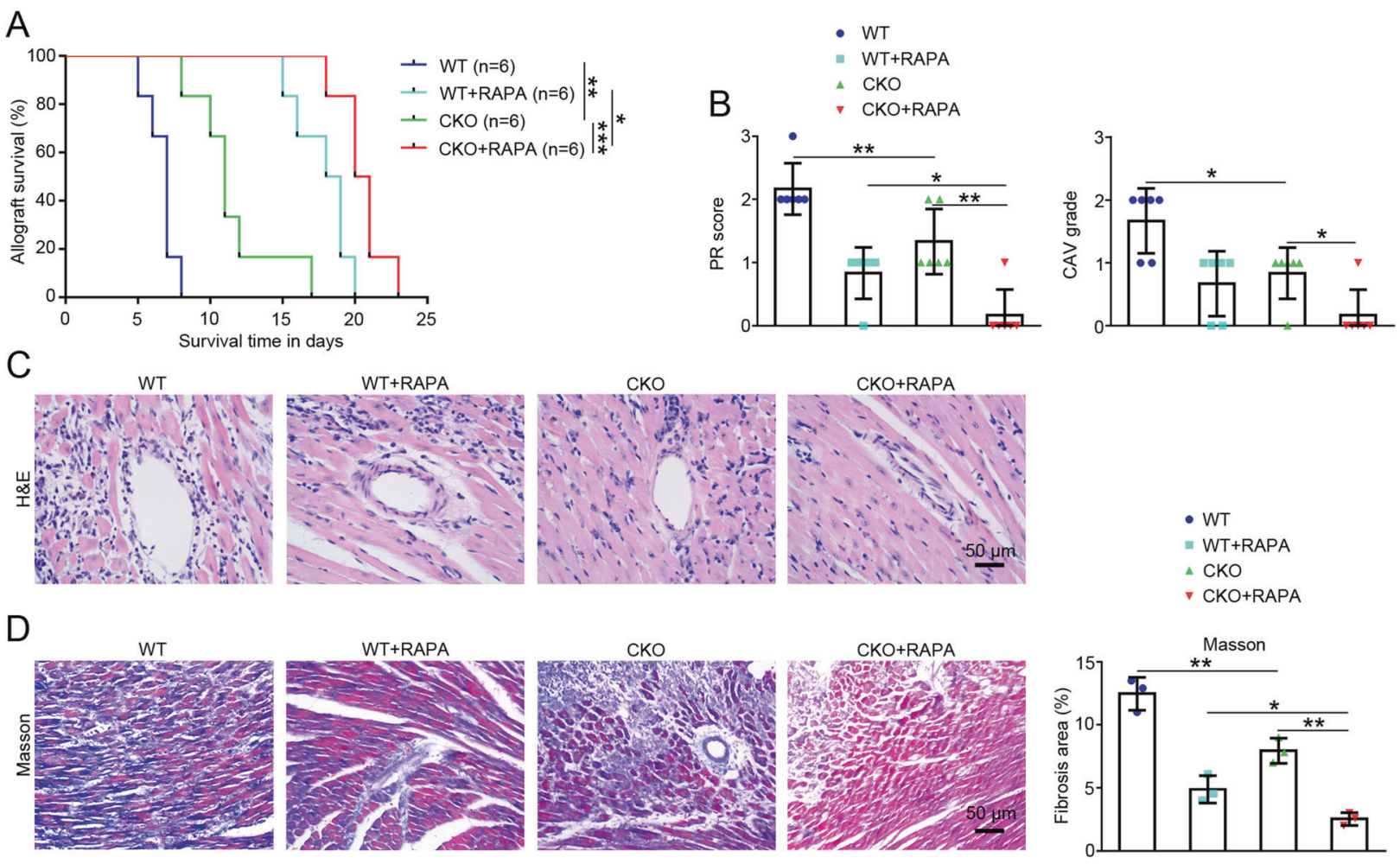

$\mathrm{E}$
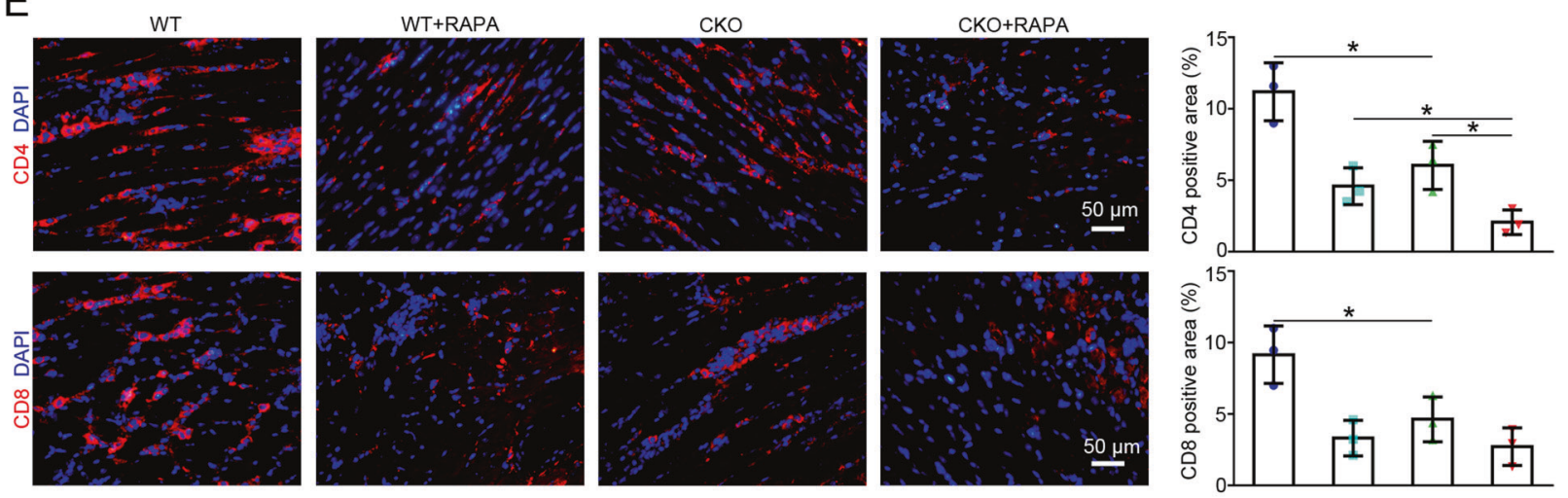

$\mathrm{F}$
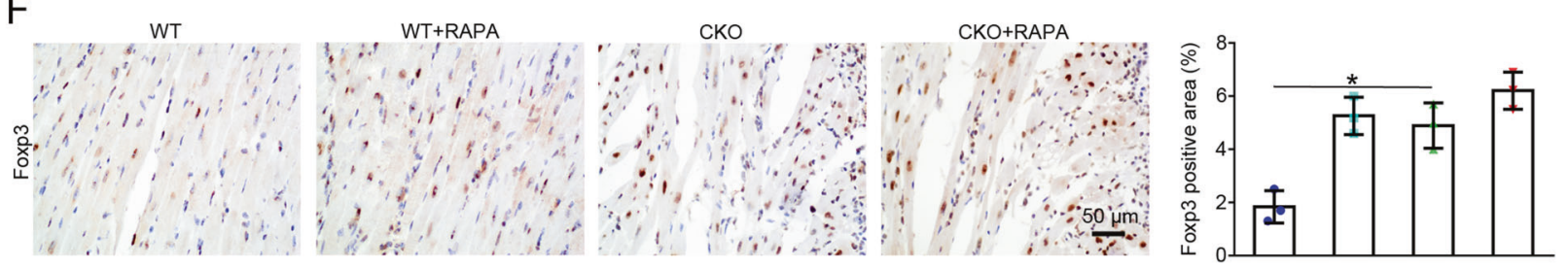

Fig. 2 The effects of the absence of miR-146a on immune rejection after heart transplantation. A Kaplan-Meier analysis of the allograft survival time (log-rank test), $n=6$. B Assessment of PR scoring and CAV grading of allografts using H\&E staining according to the ISHLT guidelines, $n=6$. C Histological analyses of the harvested allografts stained with H\&E ( $\times 400$; scale bars $=50 \mu \mathrm{m} ; n=6)$ and $\mathbf{D}$ Masson's trichrome $(\times 200$; scale bars $=50 \mu \mathrm{m} ; n=3)$ were performed on day 5 after transplantation in the WT, WT+RAPA, CKO, and CKO+RAPA groups. E The infiltration of $\mathrm{CD}^{+}$and $\mathrm{CD} 8^{+}$T cells in the allografts of the WT, WT+RAPA, CKO, and CKO+RAPA groups on day 5 were detected using immunofluorescence staining $(\times 400$; scale bars $=50 \mu \mathrm{m}), n=3$. F The infiltration of Tregs (Foxp $\left.3^{+}\right)$in the allografts of the WT, WT + RAPA, CKO, and CKO+RAPA groups on day 5 were detected using immunohistochemistry $(\times 400$; scale bars $=50 \mu \mathrm{m}), n=3$. Data are presented as means \pm SEM. ${ }^{*} p<0.05$ and ${ }^{* *} p<0.01$. CKO indicates miR-146a conditional knockout mice. WT wild type, RAPA rapamycin.

The absence of miR-146a in Tregs inhibited M1 macrophage polarization and promoted M2 macrophage polarization

\section{in vitro}

The effects of a miR-146a deficiency in Tregs on macrophage $\left(\mathrm{M} 1, \mathrm{CD} 16 / 32^{+} \mathrm{CD}^{+} 8^{+}\right.$and $\mathrm{M} 2, \mathrm{CD} 06^{+} \mathrm{CD}^{+} 8^{+}$) polarization were investigated in co-culture experiments in vitro. The proportion of each cell type was analyzed using flow cytometry. As shown in Fig. 5A, in the presence of stimuli, co-culturing monocytes with CKO Tregs significantly decreased the proportion of M1 macrophages and increased the proportion of M2 

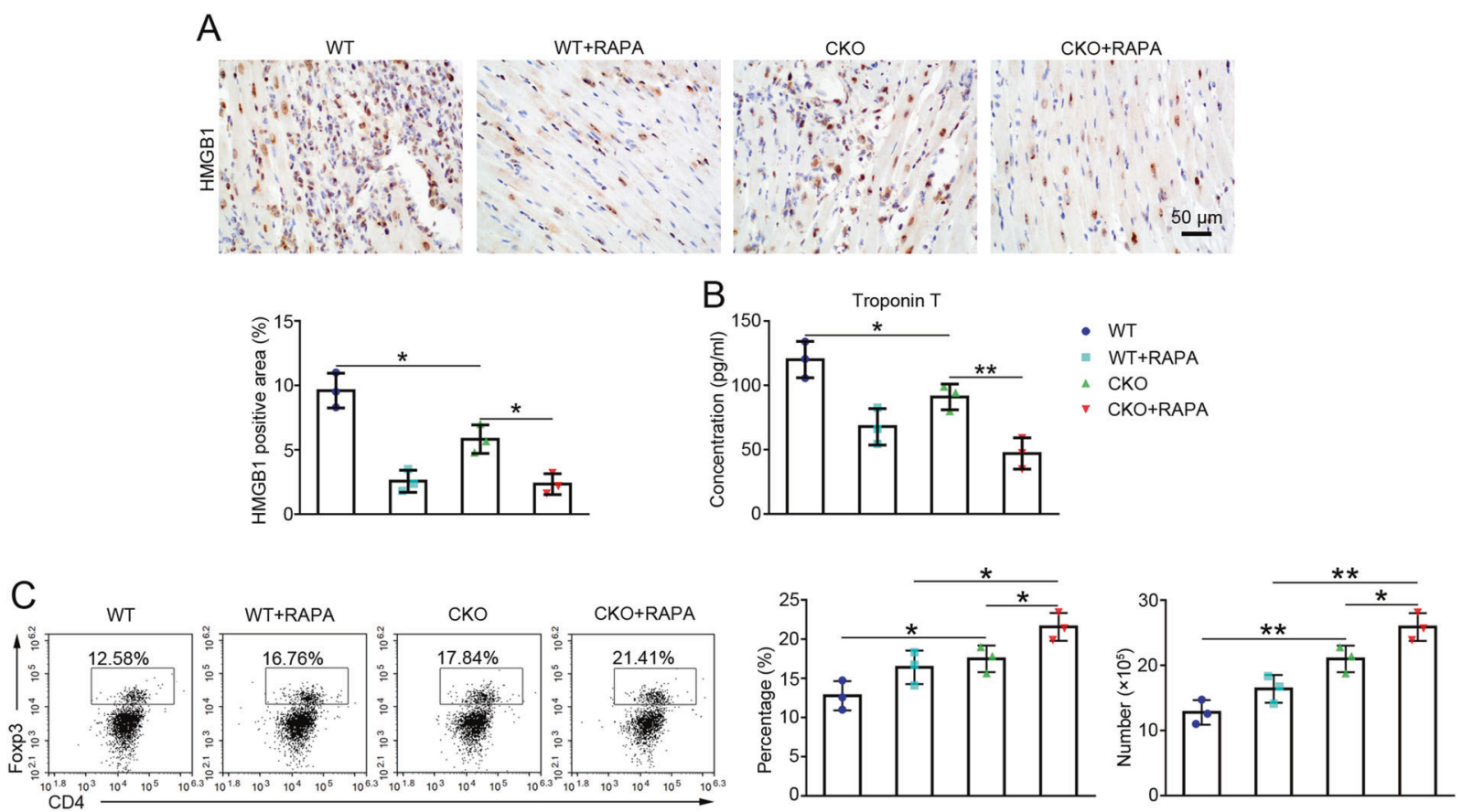

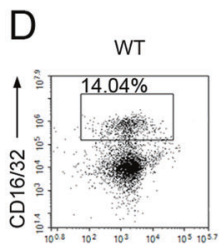

WT+RAPA
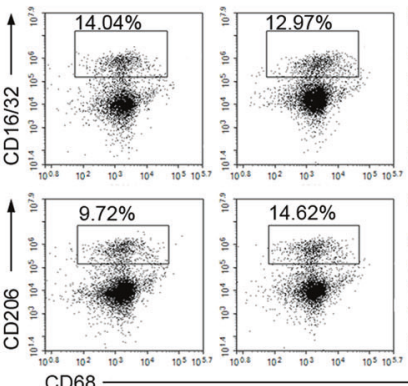

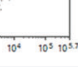

CKO
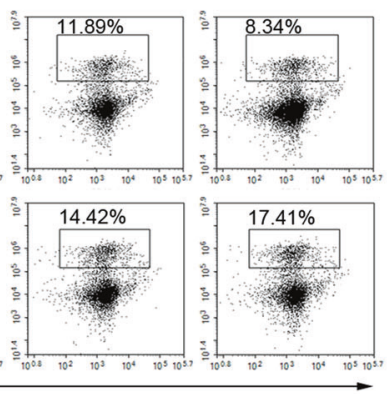

CKO+RAPA

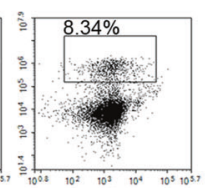

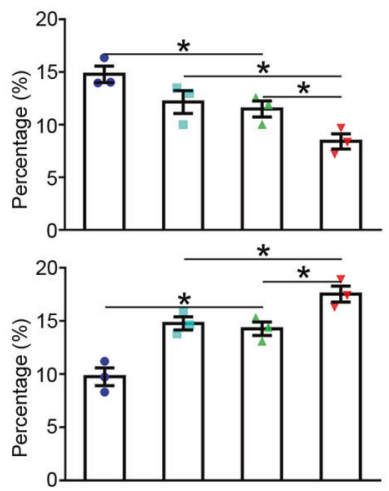

E

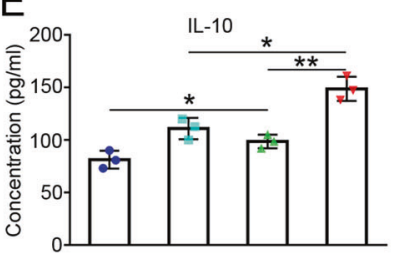

$\mathrm{F}$

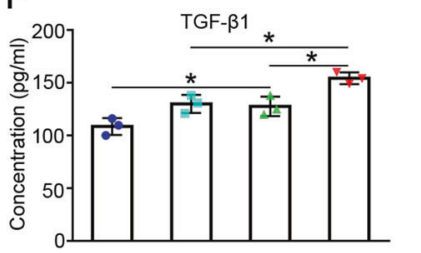

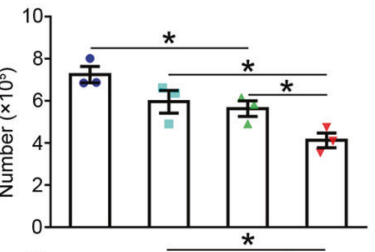

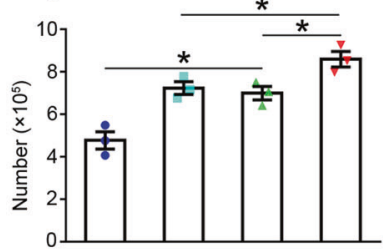

- WT

- WT+RAPA

A CKO

$\checkmark$ CKO+RAPA

Fig. 3 The effects of the absence of miR-146a on the function of allografts and immune response in recipients after heart transplantation. A The expression of HMGBI in the allografts was detected using immunohistochemistry $(\times 400 ; \mathrm{scale}$ bars $=50 \mu \mathrm{m})$ on day 5 in the WT, WT+RAPA, CKO, and CKO+RAPA groups, $n=3$. B Serum levels of Troponin T were detected in recipient mice from all groups on day 5 using an ELISA. $n=3$. C The flow cytometry analysis of the proportions and numbers of Tregs $\left(\mathrm{CD}^{+}{ }^{+}\right.$Foxp $\left.3^{+}\right)$in the spleens of recipients was performed on day 5 in all groups, $n=3$. D The flow cytometry analysis of the proportions and numbers of $M 1\left(C D 16 / 32^{+} \mathrm{CD} 68^{+}\right)$and $\mathrm{M}_{2}$ $\left(\mathrm{CD} 206^{+} \mathrm{CD}^{+} 8^{+}\right)$macrophages in the spleens of recipients was performed on day 5 in all groups, $n=3$. E-F Serum levels of IL-10 and TGF- $\beta 1$ were detected in recipients from all groups on day 5 using ELISAs, $n=3$. Data are presented as means \pm SEM. ${ }^{*} p<0.05$ and ${ }^{* *} p<0.01$. CKO indicates miR-146a conditional knockout mice. WT wild type, RAPA rapamycin.

macrophages compared with co-culturing monocytes with WT Tregs. In addition, the levels of TNF- $\alpha$ and IL-1 $\beta$ secreted by $\mathrm{M} 1$ macrophages (Fig. $5 \mathrm{~B}$ ) and IL-10 secreted by $\mathrm{M} 2$ macrophages (Fig. 5C) were decreased and increased, respectively, in the CKO group compared with the WT group in the cell co-culture system. Based on these results, the absence of miR$146 a$ enhanced the immunoregulatory effect of Tregs on macrophages.
The absence of miR-146a in Tregs regulated $\mathrm{CD}^{+} \mathrm{T}$ cell behaviors in vitro

$\mathrm{CD}^{+}{ }^{+}$cells were co-cultured with Tregs isolated from the WT or CKO group to determine the effect of miR-146a on the immunosuppressive function of Tregs. The proliferation rate of $\mathrm{CD}^{+}{ }^{+} \mathrm{T}$ cells was analyzed after co-culture with Tregs using flow cytometry. As shown in Fig. 6A, CKO Tregs significantly inhibited the proliferation of $\mathrm{CD}^{+}{ }^{+} \mathrm{T}$ cells compared with WT Tregs. 
A
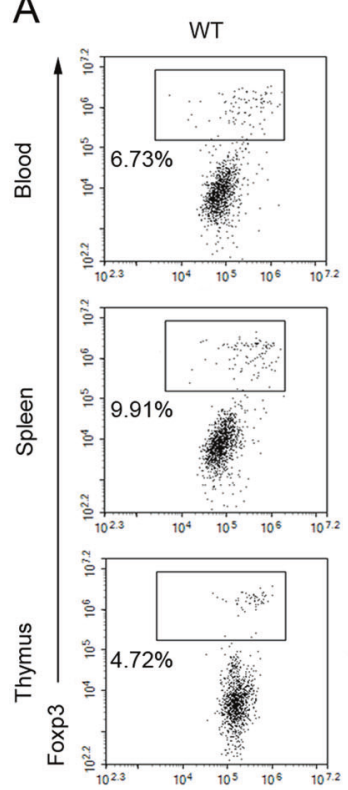

CD4
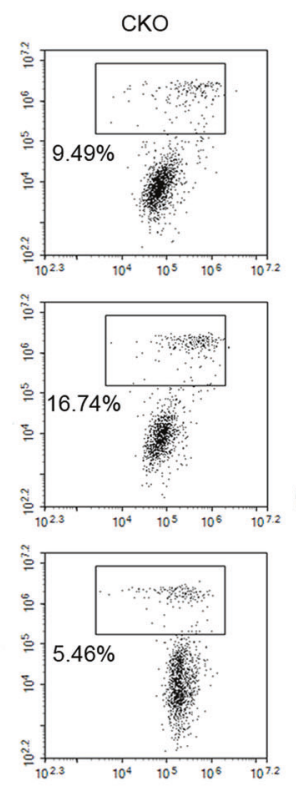

$\mathrm{B}$
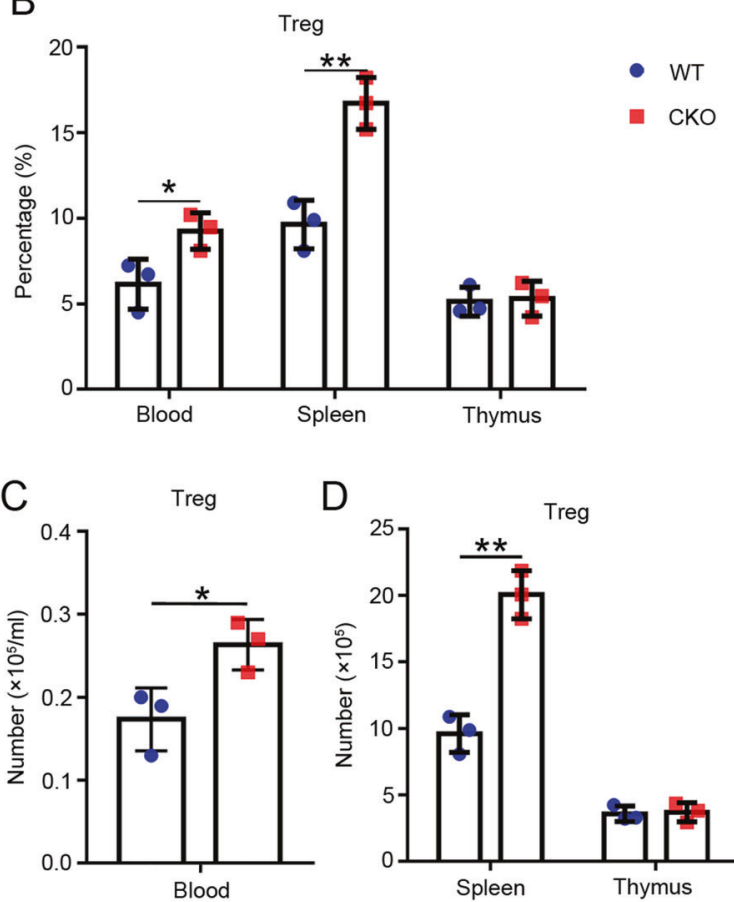

D Treg

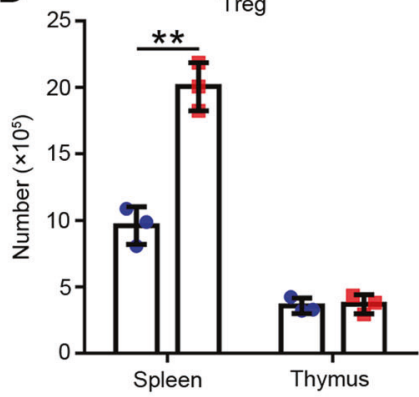

$\mathrm{E}$

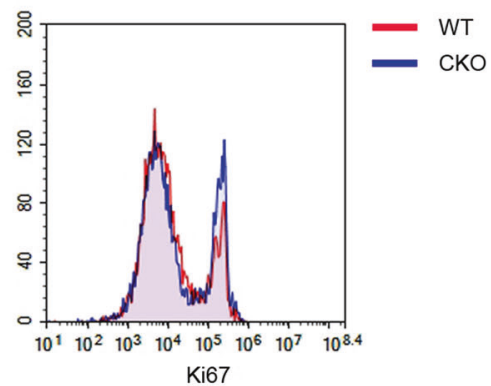

$\mathrm{F}$

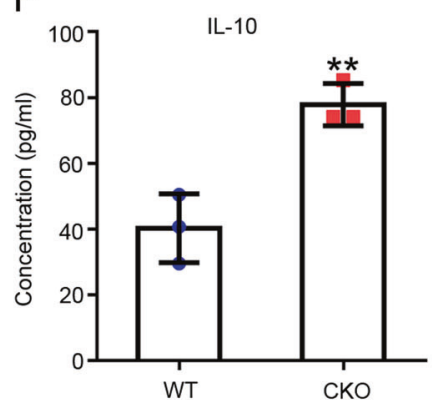

G

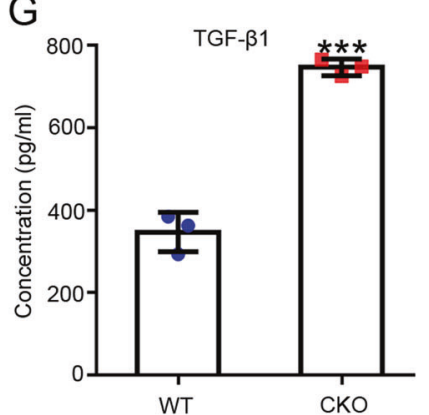

Fig. 4 The effects of the absence of miR-146a on the proportion, levels of inflammatory factors, and Ki67 expression in Tregs from mice in different groups. Flow cytometry analysis of the A-B proportions and C-D numbers of Tregs (CD4 ${ }^{+}$Foxp $\left.3^{+}\right)$in the peripheral blood, spleen, and thymus of the WT or CKO mice, $n=3$. e Flow cytometry analysis of the Ki67 expression level in Tregs isolated from the WT or CKO mouse spleens, $n=3$. F-G Serum levels of IL-10 and TGF- $\beta 1$ in WT or CKO mice were detected using ELISAs, $n=3$. Data are presented as means \pm SEM. ${ }^{*} p<0.05,{ }^{* *} p<0.01$, and ${ }^{* * *} p<0.001$. CKO indicates miR-146a conditional knockout mice. WT wild type, Treg regulatory T cell.

The apoptosis of $\mathrm{CD}^{+}{ }^{+} \mathrm{T}$ cells co-cultured with Tregs was detected using TUNEL staining. A higher apoptosis rate was observed for $\mathrm{CD}^{+} \mathrm{T}$ cells in the CKO group than in the WT group (Fig. 6B).

Moreover, the migration of $\mathrm{CD}^{+}{ }^{+} \mathrm{T}$ cells co-cultured with Tregs was evaluated by performing Transwell experiments. CXCL8 was used to induce $\mathrm{CD}^{+} \mathrm{T}$ cell migration [26]. As shown in Fig. 6C, the $\mathrm{CD} 4^{+} \mathrm{T}$ cell migration efficiency was significantly decreased in the presence of CXCL8 in the CKO group compared with the WT group.

Further analysis of the differentiation of $\mathrm{CD}^{+} \mathrm{T}$ cells cocultured with Tregs showed that the proportion of Th1 (CD4 ${ }^{+} \mathrm{IFN}$ $\mathrm{Y}^{+}$) cells (Fig. 6D) and the expression level of the Th1 signature cytokine IFN- $\gamma$ (Fig. 6E) were elevated in the CKO group compared to the WT group.

These results suggest that the absence of miR-146a does not contribute to the overall suppressive function of Tregs, but rather appears to impair the ability of Tregs to control the Th1 responses.

\section{miR-146a regulated Tregs via the IFN-p/STAT1 signaling} pathway

We measured NF-KB expression in Tregs to elucidate the mechanism by which miR-146a regulates Tregs. Levels of the TRAF6 and IRAK1 mRNAs and proteins and levels of phosphorylated IKB and p65, markers of NF-kB activation, were increased in CKO Tregs (Fig. 7A-B). In addition, levels of the TRAF6 and IRAK1 proteins and phosphorylated $\mathrm{I} K B$ and $\mathrm{p} 65$, markers of NF-KB activation, were all increased in Tregs from the CKO recipient group compared to those from the WT recipient group in animal models (Fig. 7C). Foxp3 is a lineage-specific transcription factor responsible for the differentiation and function of Tregs [27, 28]. However, Foxp3 was downregulated in CKO Tregs (Fig. 7A-B) and CKO recipient Tregs (Fig. 7C) compared with WT Tregs and WT recipient Tregs, respectively, suggesting that expression and activation of NF-KB were not responsible for Treg function.

Because a single miRNA regulates multiple targets simultaneously, we next explored the molecular mechanisms by which miR-146a-deficient Tregs mediate Th1 responses, and found that 
A
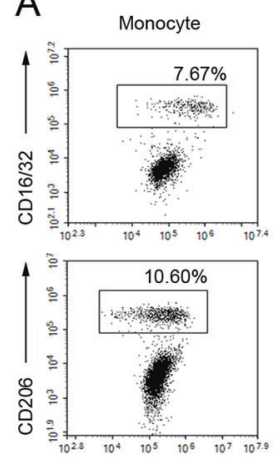

CD68
Monocyte+St
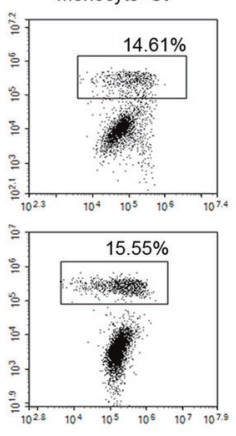

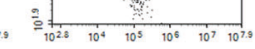
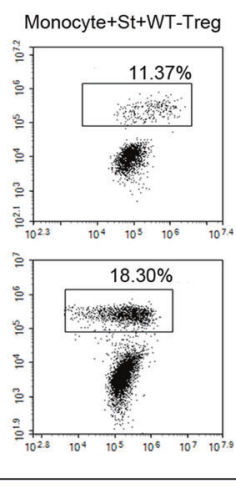
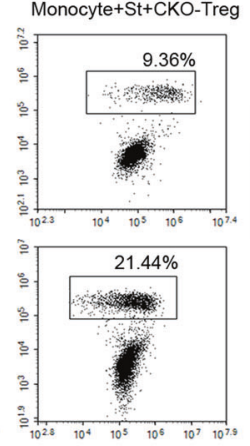

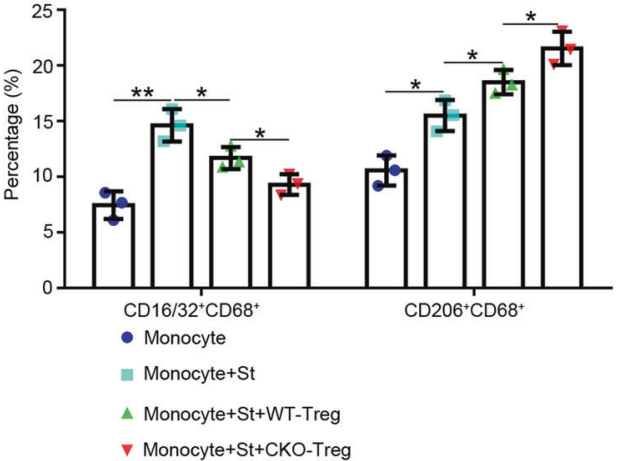

$\checkmark$ Monocyte+St+CKO-Treg

$\mathrm{B}$

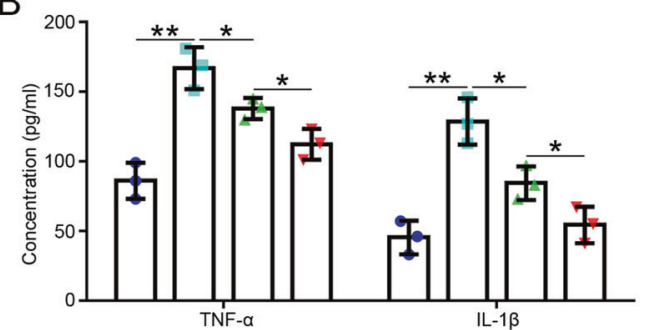

C

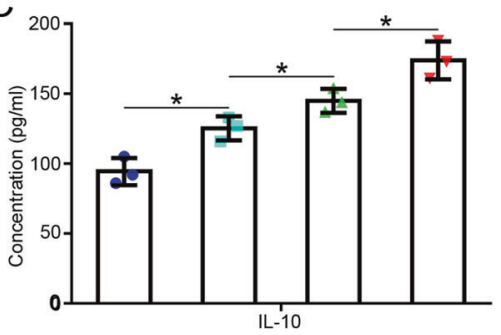

- Monocyte

- Monocyte+St

A Monocyte+St+WT-Treg

$\checkmark$ Monocyte+St+CKO-Treg

Fig. 5 The effects of the absence of miR-146a in Tregs on macrophage polarization. A Monocytes from BALB/C spleens were co-cultured with or without WT-Tregs or CKO-Tregs and different stimuli for $72 \mathrm{~h}$, and a flow cytometry analysis of the proportions of M1 (CD16/32 $\left.\mathrm{CD}^{+} 8^{+}\right)$and M2 $\left(\mathrm{CD} 206^{+} \mathrm{CD}^{+} 8^{+}\right)$macrophages was performed, $n=3$. B-C The levels of TNF- $\alpha, \mathrm{IL}-1 \beta$, and IL- 10 in the supernatant of the cocultures of monocytes and Tregs were detected using ELISAs. $n=3$. Data are presented as means \pm SEM. ${ }^{*} p<0.05$ and ${ }^{* *} p<0.01$. St indicates the corresponding stimuli. CKO indicates miR-146a conditional knockout mice. WT-Treg and CKO-Treg indicate Tregs isolated from WT and miR-146a CKO mouse spleens, respectively. WT wild type, Treg regulatory T cell.

STAT1 in the IFN- $\gamma /$ STAT1 pathway was expressed at high levels and activated in CKO Tregs (Fig. 7D-E) and CKO recipient Tregs (Fig. 7F-G) compared with WT Tregs and WT recipient Tregs, respectively.

However, when IFN- $\gamma /$ STAT1 function was inhibited by alFN- $\gamma$, the CKO Tregs + alFN $-\gamma$ significantly reduced the proportion of Th1 cells compared to CKO Tregs+control IgG in vitro (Fig. 7H), and miR-146a deficiency combined with IFN- $\gamma$ neutralization significantly prolonged allograft MST from 11 days to 23 days in vivo (MST in the CKO+ control IgG group, 11 days; MST in the CKO + alFN- $\gamma$ group, 23 days; $p<0.001$; Fig. 7l).

\section{DISCUSSION}

Immune rejection after organ transplantation is a difficult problem in immunology [29]. As our understanding of immune rejection has improved, emerging therapeutic strategies (including blocking costimulatory signals and inducing donor antigen tolerance) have entered the clinical arena [30-32]. The use of Tregs is one of the most promising natural mechanisms and strategies for inducing tolerance [33]. Given the important regulatory role of miRNAs in innate and adaptive immune cell development and function [34], our study suggested a new strategy based on miRNAs to regulate Tregs. The observed results are described below. First, the absence of miR-146a slightly inhibited rejection in animal models, and the therapeutic effect was more significant in the presence of RAPA treatment. Second, the absence of miR-146a promoted Treg expansion but impaired their function. Third, the absence of miR$146 a$ in combination with IFN- $\gamma$ treatment reversed the impaired function of Tregs and further alleviated rejection. Finally, miR-146a regulated Tregs through the IFN- $\gamma /$ STAT1 pathway.

Abnormal inhibition or activation of T lymphocytes induces an abnormal immune response; however, the recruitment and activation of $\mathrm{T}$ lymphocytes, the participation of Tregs, and their inflammatory factors are involved in immune rejection $[35,36]$. The balance between these two cell types is very important for inducing immune tolerance and moderately controlling immune rejection. Similarly, we observed changes in the Treg number and levels of anti-inflammatory factors (IL-10 and TGF- $\beta 1$ ) in response to immune signaling in the absence of miR-146a during rejection.

Macrophages are the first line of defense in tissue immune responses [37]. Differently activated macrophages (M1 and M2) exhibit different phenotypes and functions. M1 macrophages tend to secrete proinflammatory cytokines and aggravate tissue damage, while $M 2$ macrophages counteract the $M 1$ response by reducing inflammation and repairing damaged tissue [38]. Tian et al. indicated that Tregs are important inhibitors of macrophage activation [39], and subsequent studies found that Tregs induce macrophage activation in an alternative manner, as reflected by inhibiting M1 polarization while promoting M2 polarization [4042]. In this study, the absence of miR-146a enhanced the ability of Tregs to promote macrophage polarization to the $M 2$ type in vivo and in vitro and inhibited M1 macrophage polarization. Increased M2 macrophage polarization promotes the inhibition of rejection [43]. Therefore, we propose that the extent of macrophage activation is closely related to the outcome of the allografts.

The absence of miR-146a in Tregs exerted a mild suppressive effect, prolonging the allograft MST from only 7 days to 11 days in animal models. We investigated the regulatory effect of miR-146adeficient Tregs on $\mathrm{CD}^{+} \mathrm{T}$ cells in vitro to explain this phenomenon. Subsequently, in the co-cultures of Tregs and $\mathrm{CD}^{+} \mathrm{T}$ cells in vitro, the absence of miR-146a enhanced Tregmediated inhibition of $\mathrm{CD}^{+} \mathrm{T}$ cell proliferation and migration and promotion of $\mathrm{CD}^{+} \mathrm{T}$ cell apoptosis but impaired the ability of Tregs to suppress Th1 differentiation, suggesting that the absence of miR-146a is detrimental to a specific aspect of Treg suppressor function, consistent with a publication by Lu et al. [23]. Furthermore, these authors observed that miR-146a ${ }^{-1-}$ mice 
A
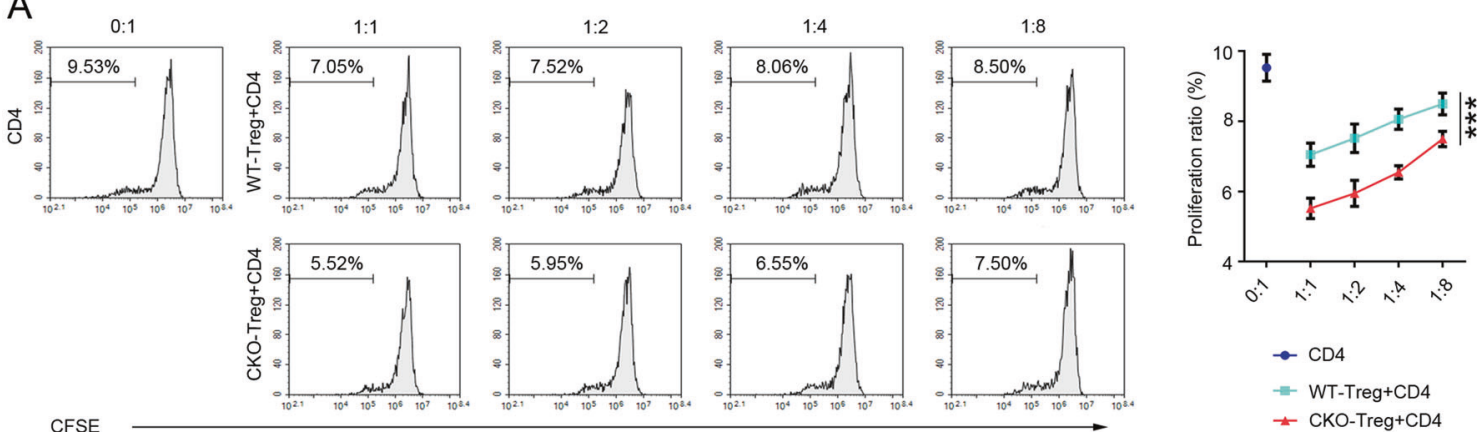

B
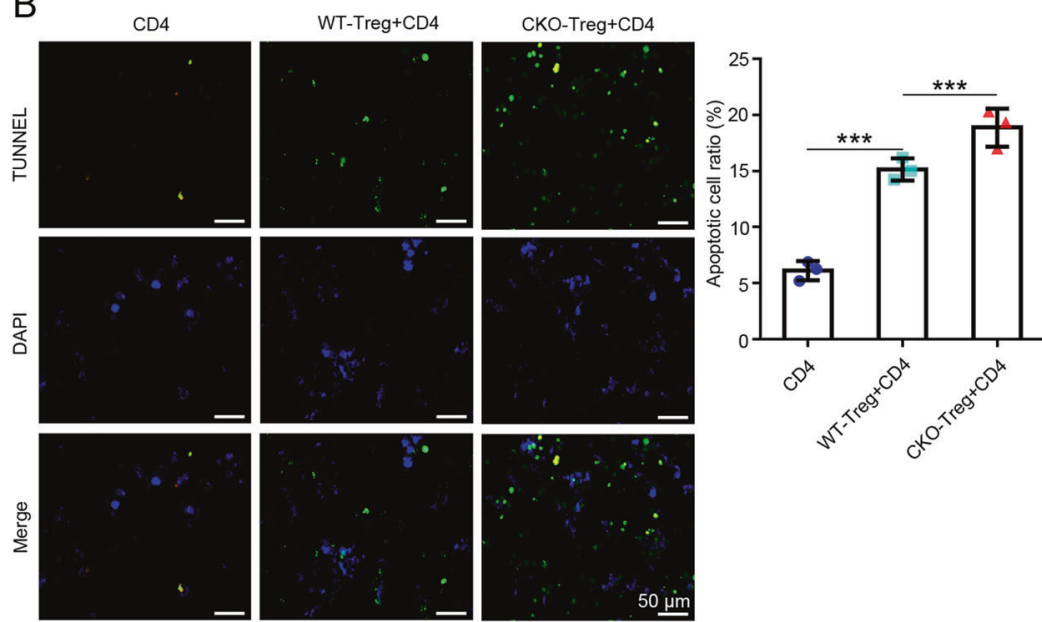

C
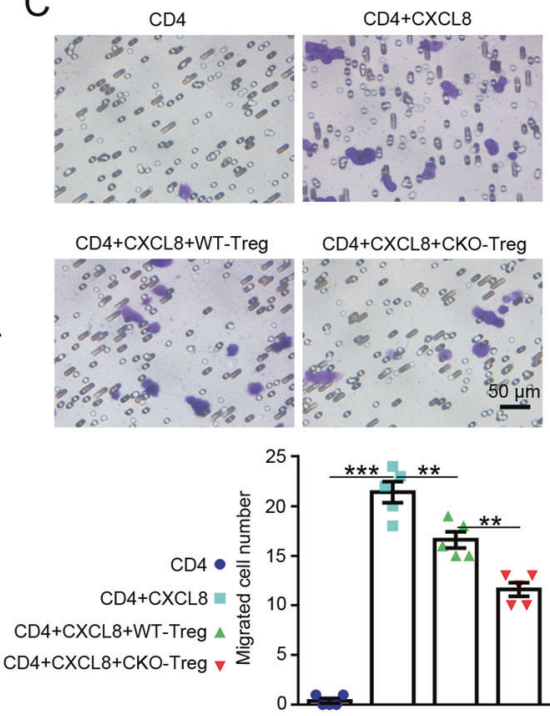

D
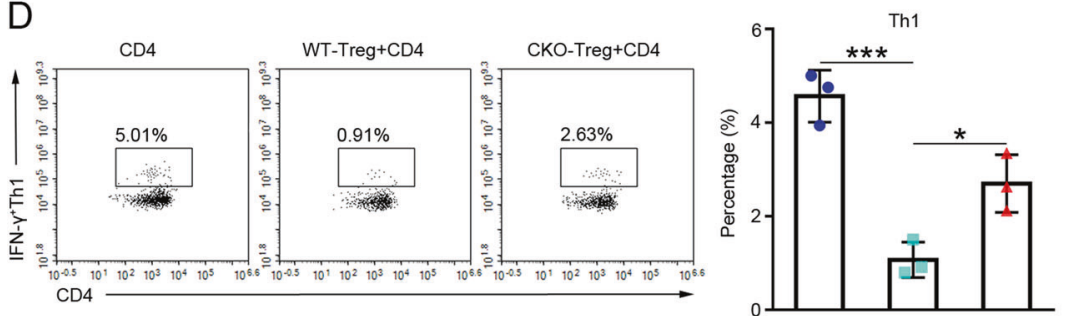

E

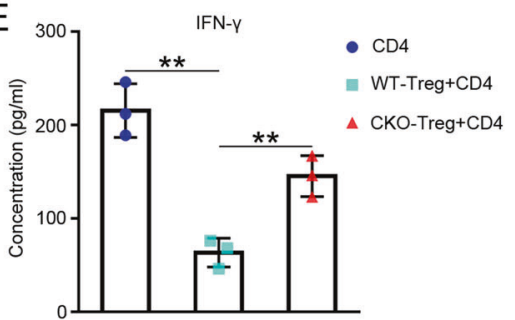

Fig. 6 The effects of the absence of miR-146a in Tregs on $\mathrm{CD4}^{+} \mathrm{T}$ cell behaviors. A Flow cytometry analysis of the proliferation rate of the CFSE-labeled CD4 ${ }^{+}$T cells cultured alone or co-cultured with WT-Tregs or CKO-Tregs for $72 \mathrm{~h}, n=3$. B Images of TUNEL staining ( $\times 400$; scale bars $=50 \mu \mathrm{m}$ ) indicating the apoptosis of $\mathrm{CD}^{+}$T cells cultured alone or co-cultured with WT-Tregs or CKO-Tregs for $72 \mathrm{~h}, n=3$. C Images of the Transwell migration assay $(\times 400$; scale bars $=50 \mu \mathrm{m})$ of the CD4 ${ }^{+} \mathrm{T}$ cells cultured alone or co-cultured with WT-Tregs or CKO-Tregs and CXCL8 for $72 \mathrm{~h}, n=5$. D Flow cytometry analysis of the differentiation of CD4 ${ }^{+}$T cell subsets (Th1) cultured alone or co-cultured with WT-Tregs or CKO-Tregs for $72 \mathrm{~h}, n=3$. E The level of IFN- $\gamma$ in the co-culture supernatant was detected using an ELISA, $n=3$. Data are presented as means \pm SEM. ${ }^{*} p<0.05,{ }^{* *} p<0.01$, and ${ }^{* * *} p<0.001$. CD4 indicates CD4 ${ }^{+}$T cells isolated from BALB/c spleens. CKO indicates miR-146a conditional knockout mice. WT-Treg and CKO-Treg indicate Tregs isolated from WT and miR-146a CKO mouse spleens, respectively. WT wild type, Treg regulatory $\mathrm{T}$ cell.

develop a pathological Th1-responsive disease, namely, a lethal breakdown of immune tolerance due to the production of large amounts of the Th1 signature cytokine IFN $-\gamma$ by miR-146adeficient $T$ cells and the failure of miR-146a-deficient Tregs to control IFN- $\gamma$ responses [23]. However, in our study, the absence of miR-146a specifically in Tregs but not in T cells avoided the effect of miR-146a on T cells in miR-146a CKO mice; therefore, further investigations are needed to determine whether CKO mice exhibit an IFN- $\gamma$-mediated Th1 pathological disease.

Foxp3 is a key regulator of Tregs that maintains Treg function and lineage stability by controlling the expression of various gene regulators, including miRNAs [44, 45]. According to previous studies, NF-KB positively influences Treg function, at least in part, by regulating the transcription of Foxp3 $[46,47]$. Liu et al. showed that Foxp3 inhibits tumor cell proliferation and promotes apoptosis by regulating the miR-146a/NF-KB axis in breast cancer and prostate cancer $[48,49]$. However, researchers have not clearly determined whether miR-146a and NF-KB are involved in regulating Tregs during rejection. In this study, NF$K B$ expression and activation were increased in miR-146adeficient Tregs, while Foxp3 expression was decreased, suggesting that miR-146a does not function through NF-KB to modulate Foxp3 expression and Treg function. In addition, the changes in Treg function in response to miR-146a deficiency may not only 

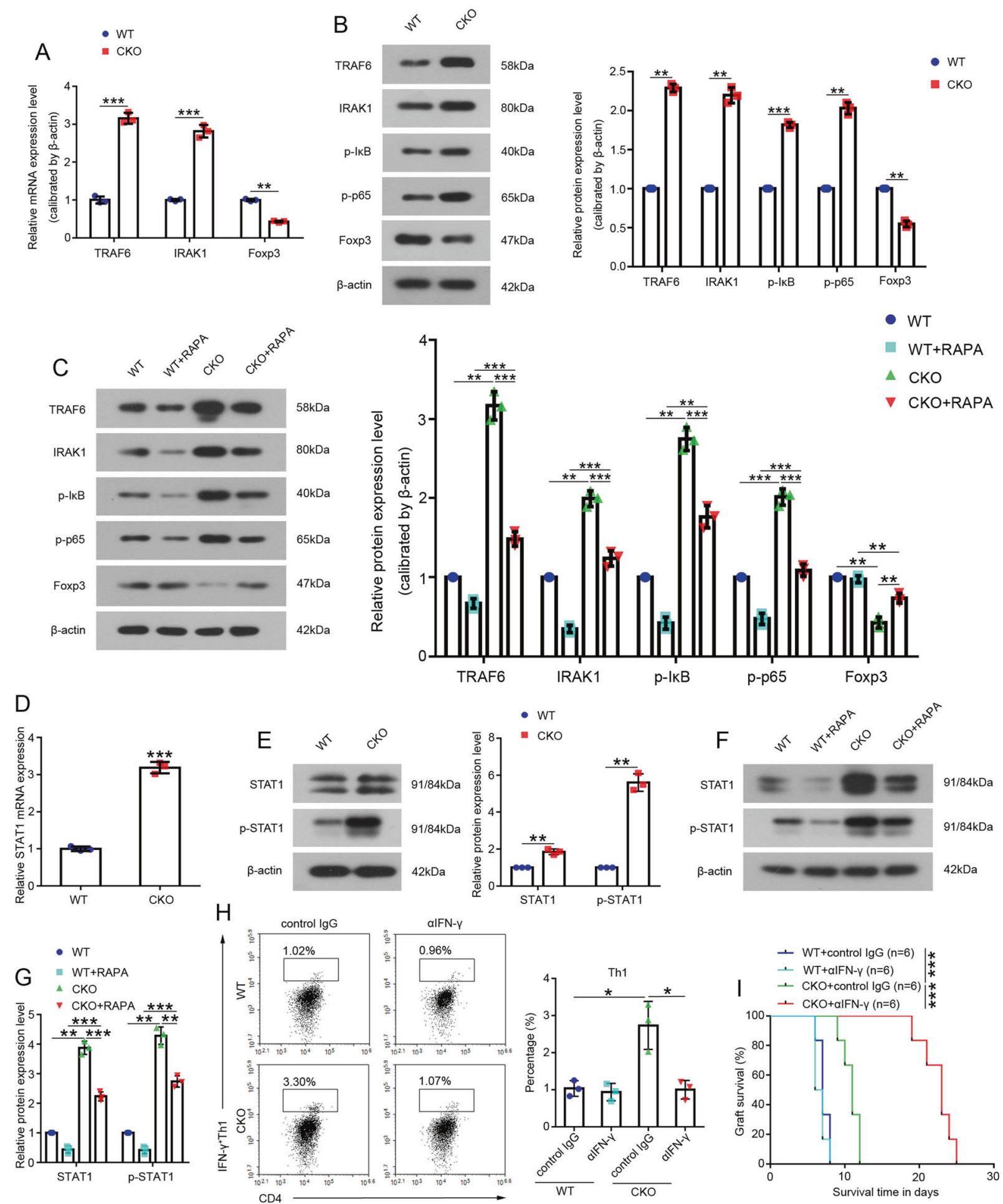

Fig. 7 The related mechanism by which miR-146a regulates Tregs. A Expression of the TRAF6, IRAK1, and Foxp3 mRNAs in Tregs isolated from the WT mouse or CKO mouse spleens, $n=3$. B Levels of the total TRAF6, IRAK1, and Foxp3 proteins and phosphorylated IKB and p65 proteins in Tregs isolated from the WT mouse or CKO mouse spleens, $n=3$. C Levels of the total TRAF6, IRAK1, and Foxp3 proteins and phosphorylated IKB and p65 proteins in Tregs isolated from recipient's spleens were detected on day 5 in the WT, WT+RAPA, CKO, and CKO + RAPA groups. $n=3$. D The levels of the mRNA and $\mathbf{E}$ total and phosphorylated STAT1 protein in Tregs isolated from the WT or CKO mouse spleens. $n=3$. F-G The total protein level and phosphorylation of STAT1 in Tregs isolated from the recipient's spleens were analyzed on day 5 in the WT, WT+RAPA, CKO, and CKO+RAPA groups, $n=3$. H CD4 ${ }^{+}$T cells from BALB/c spleens were co-cultured with Tregs isolated from the spleens of WT recipients or CKO recipients and treated with control IgG or $\alpha \mathrm{IFN}-\gamma$ for $72 \mathrm{~h}$, and then a flow cytometry analysis of the proportions of Th1 cells was performed. $n=3$. I Kaplan-Meier analysis of the allograft survival time (log-rank test). $n=6$. Data are presented as means \pm SEM. ${ }^{*} p<0.05,{ }^{* *} p<0.01$, and ${ }^{* *} p<0.001$. CKO indicates miR-146a conditional knockout mice. WT wild type, RAPA rapamycin. 
result from NF-KB but also because of the increased expression of other target genes of miR-146a, such as STAT1 [23]. In our study, we also observed increased expression and activation of the IFN- $\gamma /$ STAT1 in miR-146a-deficient Tregs, which resulted in a failure of Tregs to control the Th1 responses. When IFN- $\gamma /$ STAT1 function was inhibited, miR-146a-deficient Tregs restricted Th1 responses and prolonged allograft survival in the presence of alFN- $\gamma$. Therefore, miR-146a-mediated regulation of Tregs was mainly dependent on the IFN- $\gamma / \mathrm{STAT} 1$ rather than NF-KB during rejection. The dominance of the increased expression and activation of the IFN- $\gamma /$ STAT1 in miR-146a-deficient Tregs may have masked the effect of NF-KB.

Interestingly, the miR-146a deficiency increased the Treg number but impaired their functions. When the impaired function of miR-146a-deficient Tregs was reversed by alFN- $\gamma$, the increase in Treg number was not affected by IFN- $\gamma$ blockade [23], showing that changes in the function and number of Tregs were not uniform in the absence of miR-146a. On the other hand, in autoimmune diseases such as rheumatoid arthritis, a high frequency of Tregs is enriched in inflammation sites, but inflammatory conditions force the Treg function and phenotype to become unstable, which may accelerate autoimmune inflammation [50]. These experiments imply that Treg metabolism and suppressor functions are regulated by different molecular mechanisms.

\section{CONCLUSIONS}

In conclusion, miR-146a regulates Tregs mainly through the IFNY/STAT1 pathway during rejection in mouse heart transplantation recipients, which is closely related to the ability of Tregs to suppress Th1 responses. The absence of miR-146a in Tregs combined with IFN- $\gamma$ neutralization significantly inhibited Th1 responses, prolonged allograft survival, and alleviated rejection. This study showed the Treg-mediated post-transplant immune tolerance from the perspective of regulating a single miRNA, miR-146a, providing new insights into Treg function and a new strategy for improving the function of Tregs to suppress clinical rejection.

\section{MATERIALS AND METHODS}

\section{Animals}

Wild-type C57BL/6 (WT) and BALB/c mice weighing 20-25 g and aged 6 to 8 weeks were purchased from the Experimental Animal Center of the Chinese Academy of Medical Sciences (Beijing, China). The miR-146a flox/flox

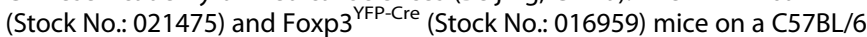
background were purchased from The Jackson Laboratory (Bar Harbor, ME, USA). For the generation of miR-146a-conditional knockout mice (CKO; Fig. S1), miR-146a ${ }^{\text {flox/flox }}$ and Foxp3 $3^{\text {YFP-Cre }}$ mice were interbred as described in a previous report [51]. All animals were matched for sex and age, randomly assigned to each group, and included in the analysis. All the experimental procedures followed protocols approved by the Animal Care and Use Committee of Tianjin Medical University General Hospital (Ethical Approval No.: IRB2015-YX-009).

\section{Mouse heart transplantation model}

Intraabdominal heterotopic cardiac transplantation was performed as previously described [52]. Briefly, donor hearts from WT or BALB/c mice were transplanted into WT or CKO mouse recipients by microsurgery. In the isogeneic transplant group, both the donor and recipient were WT mice. The donor in the allogeneic group was a BALB/c mouse and the recipient was WT or CKO mouse. Then, all allogeneic transplant recipients were randomly divided into 4 groups $(n=6)$ as follows: $\mathrm{BALB} / \mathrm{c} \rightarrow \mathrm{WT}$, $\mathrm{BALB} / \mathrm{c} \rightarrow \mathrm{WT}+$ rapamycin (RAPA), BALB/C $\rightarrow \mathrm{CKO}$, and $\mathrm{BALB} / \mathrm{C} \rightarrow \mathrm{CKO}$ + RAPA. Some recipient groups were subcutaneously injected with RAPA at a dose of $2 \mathrm{mg}$ daily for 13 days. Allograft survival was assessed daily by palpation. The sera, spleens, and allografts of the recipients were collected on the 5th day after transplantation. Tregs from the recipient's spleens were sorted using the $\mathrm{CD} 4{ }^{+} \mathrm{CD} 25^{+}$Regulatory T Cell Isolation Kit
(Miltenyi, Bergisch Gladbach, Germany) on the 3rd, 5th, and 7th days after transplantation.

\section{Evaluation of heart allograft rejection}

Allografts from the recipients of each group were collected, fixed with $4 \%$ formalin, dehydrated, embedded in paraffin, and cut into 4- $\mathrm{mm}$ sections. Histological changes were observed using hematoxylin-eosin $(\mathrm{H} \& \mathrm{E}$; Solarbio, Beijing, China) staining, and the myocardial fibrosis area was observed using Masson's trichrome staining (Solarbio). Based on H\&E staining, parenchymal rejection (PR) [53] and cardiac allograft vasculopathy (CAV) [54] of the allografts were graded according to the guidelines recommended by the International Society for Heart and Lung Transplantation (ISHLT).

For immunofluorescence staining, the sections were hydrated and blocked with normal goat serum. After incubation with the primary antibodies against CD4 (1:100; Catalog No.: ab183685; Abcam, UK) and CD8 (1:100; Catalog No.: 14-0081-82; eBioscience, USA) at $4{ }^{\circ} \mathrm{C}$ overnight, the sections were incubated with a fluorescent secondary antibody at room temperature and observed under a fluorescence microscope. The sections were evaluated by two pathologists in a double-blind manner.

For immunohistochemistry, sections were dewaxed, hydrated, subjected to antigen retrieval, and blocked with normal goat serum at room temperature for $15 \mathrm{~min}$. After incubation with primary antibodies against Foxp3 (1:200; Catalog No.: ab215206; Abcam, UK) and HMGB1 (1:200; Catalog No.: 6893; CST, USA) at $4{ }^{\circ} \mathrm{C}$ overnight, the sections were incubated with a biotinylated secondary antibody and stained with DAB substrate. After counterstaining with hematoxylin, all sections were observed under an optical microscope.

The serum level of Troponin $T$ in recipients was determined using enzyme-linked immunosorbent assay (ELISA) kits (Reddot Biotech, Canada) according to the manufacturer's instructions.

\section{Evaluation of the immune response in recipient mice}

The spleens were collected from the recipients in each group, ground, and filtered through a 40-mesh screen. Red blood cells were lysed with red blood cell lysis buffer (Solarbio), and then the remaining cells were washed twice and resuspended in phosphate-buffered saline (PBS; Solarbio). Fluorescent dye-conjugated antibodies were added to $10^{5}$ cells in $100 \mu \mathrm{l}$ of PBS and incubated in the dark for $30 \mathrm{~min}$. The following antibodies were used for the flow cytometry analysis: anti-CD4-FITC (Catalog No.: 100405; BioLegend, USA), anti-Foxp3-PE (Catalog No.: 126403; BioLegend, USA), anti-CD16/32-FITC (Catalog No.: 101305; BioLegend, USA), anti-CD206-FITC (Catalog No.: 141703; BioLegend, USA), and anti-CD68-APC (Catalog No.: 137007; BioLegend, USA) antibodies.

Serum levels of interleukin (IL)-10 and transforming growth factor (TGF)- $\beta 1$ in recipients were determined using ELISA kits (DAKEWE, Beijing, China) according to the manufacturer's instructions.

\section{Assessment of the immune microenvironment}

The spleen, peripheral blood, and thymus of WT and CKO mice were collected, and cell suspensions were prepared. Next, the cells were labeled with anti-CD4-FITC (Catalog No.: 100405; BioLegend, USA) and anti-Foxp3PE (Catalog No.: 126403; BioLegend, USA) antibodies. The percentage of positive cells was measured using flow cytometry.

Serum levels of IL-10 and TGF- $\beta 1$ were determined using ELISA kits (DAKEWE) according to the manufacturer's instructions.

\section{Isolation of Tregs and evaluation of Ki67 expression}

Tregs from WT and CKO mouse spleens were sorted using a CD4 ${ }^{+} \mathrm{CD} 25^{+}$ Regulatory T Cell Isolation Kit (Miltenyi) and labeled with the AlexaFluor ${ }^{\circledR} 488$ anti-mouse/human Ki67 antibody (Catalog No.: 151204; BioLegend, USA). The expression level of Ki67 in Tregs was measured using flow cytometry.

\section{Evaluation of macrophage polarization in vitro}

Monocytes from BALB/C spleen samples were sorted using a Mouse Monocyte Isolation Kit (Miltenyi), co-cultured with Tregs at monocyte:Treg ratios of 2:0 and 2:1, and exposed to different stimuli to determine the effect of a miR-146a deficiency in Tregs on macrophage polarization. Macrophage type 1 (M1) cells were stimulated with lipopolysaccharide (LPS; $10 \mu \mathrm{g} / \mathrm{ml}$; Solarbio) + interferon (IFN)- $\gamma(20 \mathrm{ng} / \mathrm{ml}$; PeproTech), and macrophage type 2 (M2) cells were stimulated with LPS $(10 \mu \mathrm{g} / \mathrm{ml}$; 
Solarbio) $+\mathrm{IL}-4$ ( $50 \mathrm{ng} / \mathrm{ml}$; PeproTech). After $72 \mathrm{~h}$ of co-culture, the cells from each group were harvested and labeled with anti-CD16/32-FITC (Catalog No.: 101305; BioLegend, USA), anti-CD206-FITC (Catalog No.: 141703; BioLegend, USA), and anti-CD68-APC (Catalog No.: 137007; BioLegend, USA) antibodies, and the ratios of various types of cells were detected using flow cytometry.

The levels of tumor necrosis factor (TNF)- $\alpha, \mathrm{IL}-1 \beta$, and IL-10 in the supernatant of co-cultured monocytes and Tregs were determined using ELISA kits (DAKEWE) according to the manufacturer's instructions.

\section{Isolation of $\mathrm{CD4}^{+} \mathrm{T}$ cells and stimulation with $\mathrm{CD} 3 / \mathrm{CD} 28$ microbeads}

$\mathrm{CD}^{+} \mathrm{T}$ cells from BALB/c spleens were sorted with the mouse CD4 (L3T4) MicroBeads Isolation Kit (Miltenyi). The purified $\mathrm{CD}^{+}{ }^{+} \mathrm{T}$ cells were labeled with carboxyfluorescein succinimidyl amino ester (CFSE) $(100 \mu \mathrm{g} / \mathrm{ml}$; BD Biosciences, CA, USA). $\mathrm{CD}^{+} \mathrm{T}$ cells were stimulated with $\mathrm{CD} 3 / \mathrm{CD} 28$ microbeads (bead-to-cell ratio of 1:1; Miltenyi) for $72 \mathrm{~h}$.

\section{Evaluation of $\mathrm{CD4}^{+} \mathrm{T}$ cell behaviors in vitro}

We performed lymphocyte co-culture experiments to examine the effect of the absence of miR-146a in Tregs on $\mathrm{CD}^{+} \mathrm{T}$ cell behaviors. For the $\mathrm{CD}^{+}$ $\mathrm{T}$ cell proliferation assay, Tregs and CFSE-labeled $\mathrm{CD}^{+} \mathrm{T}$ cells were cocultured for $72 \mathrm{~h}$ at Treg: $\mathrm{CD} 4^{+} \mathrm{T}$ cell ratios of 0:1, 1:1, 1:2, 1:4, and 1:8, and the proliferation response of $\mathrm{CD}^{+}{ }^{+} \mathrm{T}$ cells was assessed using flow cytometry.

For the $\mathrm{CD} 4^{+} \mathrm{T}$ cell apoptosis assay, Tregs and $\mathrm{CD} 4^{+} \mathrm{T}$ cells were seeded at Treg:CD4 ${ }^{+} \mathrm{T}$ cell ratios of 0:1 and 1:1 into the upper and lower chambers of $0.4-\mu \mathrm{m}$ polycarbonate membrane Transwell inserts (Corning, NY, USA), respectively. After $72 \mathrm{~h}$ of co-culture, $\mathrm{CD}^{+} \mathrm{T}$ cells were harvested and cultured on 13 -mm-diameter glass coverslips in 24 -well plates at $37^{\circ} \mathrm{C}$ for $15 \mathrm{~min}$, and then the cells were permeabilized with $0.1 \%$ Triton X-100 (Beyotime, Shanghai, China) for $15 \mathrm{~min}$ at room temperature. After these basic procedures, the cells were subjected to TUNEL (Roche, Basel, Switzerland) staining according to the manufacturer's instructions, and images were obtained with a confocal microscope (Olympus, Tokyo, Japan).

For the $\mathrm{CD}^{+}{ }^{+} \mathrm{T}$ cell migration assay, after the Tregs and $\mathrm{CD} 4^{+} \mathrm{T}$ cells were co-cultured at Treg:CD4 ${ }^{+} \mathrm{T}$ cell ratios of $0: 1$ and 1:1 in a $0.4-\mu \mathrm{m}$ Transwell culture system for $72 \mathrm{~h}$ as described above, $\mathrm{CD}^{+}$T cells translocated to the upper chambers of $8-\mu \mathrm{m}$ polycarbonate membrane Transwell inserts (Corning). RPMI-1640 medium containing CXCL8 (150 ng/ $\mathrm{ml}$; PeproTech) was added to the lower chamber. After culturing for $4 \mathrm{~h}$, the medium was discarded, and the cells were stained with crystal violet (Amresco, Solon, OH, USA) and imaged with a microscope (Olympus). The number of migrated cells was determined in the images.

For the $\mathrm{CD}^{+}{ }^{+} \mathrm{T}$ cell differentiation assay, Tregs and $\mathrm{CD} 4^{+} \mathrm{T}$ cells were cocultured for $72 \mathrm{~h}$ at Treg:CD4 ${ }^{+} \mathrm{T}$ cell ratios of $0: 1$ and 1:1. The cells were harvested and labeled with anti-CD4-APC (Catalog No.: 100411; BioLegend, USA), anti-IFN- - -PE (Catalog No.: 505807; BioLegend, USA) antibodies, and the ratios of $\mathrm{CD} 4^{+} \mathrm{IFN}-\gamma^{+}$Th1 cells were detected using flow cytometry.

According to the manufacturer's instructions, the expression level of IFN$\gamma$ in the co-culture supernatant was detected using ELISA kits (DAKEWE).

\section{Reverse transcription-polymerase chain reaction (RT-PCR)}

Total RNA was extracted with the TRlpure kit (BioTeke, Beijing, China) and the concentration of total RNA was measured using a NANO 2000 spectrophotometer (Thermo Fisher Scientific, Waltham, MA, USA). The cDNA templates were synthesized using the Super M-MLV Reverse Transcriptase Kit (BioTeke) and amplified with the SYBR Green method in an Exicycler ${ }^{\mathrm{TM}}$ 96 Fluorescent Quantitative Analyzer (Bioneer, Daejeon, Korea). The primer sequences were as follows: miR-146a, forward: 5-TGAGAACTGAATTCCAT GGGTT-3 and reverse: 5-GCAGGGTCCGAGGTATTC-3; Foxp3, forward: 5-AG TGCTITGTGCGAGTGG-3 and reverse: 5-AAGGGCAGGGATTGGAG-3; TRAF6, forward: 5-GCAGAGGAATCACTTGGCACGAC-3 and reverse: 5-ATCGCACGG ACGCAAAGCA-3; IRAK1, forward: 5-ACAGAGGTGGAACAGCTATCA-3 and reverse: 5-TGGGCAAGAAGCCATAAAC-3; STAT1, forward: 5-CACGCCTITG GGAAGTATTA-3 and reverse: 5-GAAGCAGGTTGTCTGTGGTCT-3; U6, forward: 5-CGCAAGGATGACACGCAAAT-3 and reverse: 5-GCAGGGTCCGAGGT ATTC-3; and $\beta$-actin, forward: 5-CTGTGCCCATCTACGAGGGCTAT-3 and reverse: 5-TTTGATGTCACGCACGATTCC-3.

\section{Western blot assay}

Total protein was obtained using RIPA lysis buffer, and approximately $30 \mu \mathrm{g}$ of total protein in each sample were separated on SDS/PAGE gels in an electrophoresis system (Bio-Rad, Mini-Protein Tetra System) and transferred to a PVDF membrane (Millipore, Billerica, MA, USA). The membrane was blocked with a TBST (TBS/0.15\% Tween 20) solution containing $5 \%$ skim milk powder at room temperature for $1 \mathrm{~h}$, the solution was discarded, and the PVDF membrane was washed three times with the TBST solution, followed by overnight probing at $4{ }^{\circ} \mathrm{C}$ with anti-Foxp 3 (1:1000; Catalog No.: ab215206; Abcam, UK), anti-TRAF6 (1:5000; Catalog No.: ab33915; Abcam, UK), anti-IRAK1 (1:1000; Catalog No.: ab238; Abcam, UK), anti-p-IKB (1:1000; Catalog No.: 2859T; CST, USA), anti-p-p65 (1:1000; Catalog No.: 3033T; CST, USA), anti-STAT1 (1:1000; Catalog No.: 14994S; CST, USA), anti-p-STAT1 (1:1000; Catalog No.: 7649S; CST, USA), and anti$\beta$-actin (1:5000; Catalog No.: 4970T; CST, USA) antibodies. The membrane was rinsed, incubated with diluted horseradish peroxidase (HRP)-labeled secondary antibody (1:50000; Catalog No.: 7071T; CST, USA) for $45 \mathrm{~min}$ at room temperature, and immunoreactive protein bands were detected using an enhanced chemiluminescence assay kit (Millipore). Images were acquired with a G-Box system (Syngene, Frederick, MD, USA) and analyzed with ImageJ software.

\section{Evaluation of IFN- $\gamma$ neutralization}

For this experiment, $0.5 \mathrm{mg}$ of IFN- $\gamma$ neutralizing antibody (aIFN- $\gamma$; Catalog No.: BE0055; BioXCell, USA) or isotype-matched control IgG was injected into WT recipient or CKO recipient mice via the tail vein 1 day before and 1 and 4 days after transplantation. Tregs from the recipient's spleens were sorted using a CD4 + CD25 + Regulatory T Cell Isolation Kit (Miltenyi) on the 5th day after transplantation and co-cultured with $\mathrm{CD} 4^{+} \mathrm{T}$ cells from BALB/c spleens at Treg:CD4 ratio of 1:1. After $72 \mathrm{~h}$ of co-culture, the cells were harvested and labeled with anti-CD4-APC (Catalog No.: 100411; BioLegend, USA) and anti-IFN- $\gamma$-PE (Catalog No.: 505807; BioLegend, USA) antibodies, and the ratios of Th1 cells were detected using flow cytometry. Allograft survival was assessed daily by palpation.

\section{Flow cytometry}

Cell surface and intracellular staining assays were performed as previously described [55]. Cell fluorescence was detected using a NovoCyte Flow cytometer (ACEA Bioscience, San Diego, CA, USA), and data were analyzed using NovoExpress software (ACEA Bioscience).

\section{Statistical analysis}

The investigator and the outcome assessment were blinded during the experiment. SPSS 22.0 (IBM SPSS Statistics, Armonk, NY, USA) was used for statistical analyses. All data are presented as the means \pm standard errors of the means (mean $\pm S E M)$, and comparisons between groups were performed using a two-sided Student's $t$-test. The differences among the groups were analyzed using one-way ANOVA. Kaplan-Meier survival curves were used to analyze allograft survival. $P$ values $<0.05$ were considered statistically significant. All experiments were performed three times, and the sample size of mice was $n=3-6$ per group.

\section{REFERENCES}

1. Chen Y, Zhang L, Liu J, Zhang P, Chen X, Xie M. Molecular imaging of acute cardiac transplant rejection: animal experiments and prospects. Transplantation. 2017:101:1977-86

2. Levitsky J, Asrani SK, Schiano T, Moss A, Chavin K, Miller C, et al. Clinical trials in organ transplantation- $\mathrm{C}$ discovery and validation of a novel blood-based molecular biomarker of rejection following liver transplantation. Am J Transplant. 2020;20:2173-83.

3. Liu J, Chen Y, Wang G, Jin Q, Sun Z, Lv Q, et al. Improving acute cardiac transplantation rejection therapy using ultrasound-targeted FK506-loaded microbubbles in rats. Biomater Sci. 2019;7:3729-40.

4. Peters DH, Fitton A, Plosker GL, Faulds D. Tacrolimus: a review of its pharmacology, and therapeutic potential in hepatic and renal transplantation. Drugs. 1993;46:746-94.

5. Scott LJ, McKeage K, Keam SJ, Plosker GL. Tacrolimus: a further update of its use in the management of organ transplantation. Drugs. 2003;63:1247-97.

6. Josefowicz SZ, Lu LF, Rudensky AY. Regulatory T cells: mechanisms of differentiation and function. Annu Rev Immunol. 2012;30:531-64.

7. Barbi J, Pardoll D, Pan F. Treg functional stability and its responsiveness to the microenvironment. Immunol Rev. 2014;259:115-39.

8. Sakaguchi S, Wing K, Onishi Y, Prieto-Martin P, Yamaguchi T. Regulatory $T$ cells: how do they suppress immune responses? Int Immunol. 2009;21:1105-11. 
9. Cabello-Kindelan C, Mackey S, Sands A, Rodriguez J, Vazquez C, Pugliese A, et al. Immunomodulation followed by antigen-specific Treg infusion controls islet autoimmunity. Diabetes. 2020;69:215-27.

10. Brunstein CG, Miller JS, Cao Q, McKenna DH, Hippen KL, Curtsinger J, et al. Infusion of ex vivo expanded $\mathrm{T}$ regulatory cells in adults transplanted with umbilical cord blood: safety profile and detection kinetics. Blood. 2011;117:1061-70.

11. Scheinecker C, Goschl L, Bonelli M. Treg cells in health and autoimmune diseases: new insights from single cell analysis. J Autoimmun. 2020;110:102376.

12. Wing JB, Tanaka A, Sakaguchi S. Human FOXP3(+) regulatory T cell heterogeneity and function in autoimmunity and cancer. Immunity. 2019;50:302-16.

13. Esensten JH, Muller YD, Bluestone JA, Tang Q. Regulatory T-cell therapy for autoimmune and autoinflammatory diseases: the next frontier. J Allergy Clin Immunol. 2018;142:1710-8.

14. Ratnasothy K, Jacob J, Tung S, Boardman D, Lechler RI, Sanchez-Fueyo A, et al. IL2 therapy preferentially expands adoptively transferred donor-specific Tregs improving skin allograft survival. Am J Transplant. 2019;19:2092-100.

15. Lu Y, Hippen KL, Lemire AL, Gu J, Wang W, Ni X, et al. miR-146b antagomirtreated human Tregs acquire increased GVHD inhibitory potency. Blood. 2016;128:1424-35.

16. Sun $Q$, Yang $Z$, Li $P$, Wang $X$, Sun $L$, Wang $S$, et al. A novel miRNA identified in GRSF1 complex drives the metastasis via the PIK3R3/AKT/NF-kappaB and TIMP3/ MMP9 pathways in cervical cancer cells. Cell Death Dis. 2019;10:636.

17. Chong MM, Rasmussen JP, Rudensky AY, Littman DR. The RNAselll enzyme Drosha is critical in T cells for preventing lethal inflammatory disease. J Exp Med. 2008;205:2005-17.

18. Liston A, Lu LF, O'Carroll D, Tarakhovsky A, Rudensky AY. Dicer-dependent microRNA pathway safeguards regulatory $T$ cell function. J Exp Med. 2008;205:1993-2004.

19. Zhou X, Jeker LT, Fife BT, Zhu S, Anderson MS, McManus MT, et al. Selective miRNA disruption in $T$ reg cells leads to uncontrolled autoimmunity. J Exp Med. 2008;205:1983-91.

20. Cobb BS, Hertweck A, Smith J, O'connor E, Graf D, Cook T, et al. A role for Dicer in immune regulation. J. Exp. Med. 2006;203:2519-27.

21. Su YL, Wang X, Mann M, Adamus TP, Wang D, Moreira DF, et al. Myeloid celltargeted miR-146a mimic inhibits NF-kappaB-driven inflammation and leukemia progression in vivo. Blood. 2020;135:167-80.

22. Mann M, Mehta A, Zhao JL, Lee K, Marinov GK, Garcia-Flores $Y$, et al. An NFkappaB-microRNA regulatory network tunes macrophage inflammatory responses. Nat Commun. 2017;8:851.

23. Lu LF, Boldin MP, Chaudhry A, Lin LL, Taganov KD, Hanada T, et al. Function of miR-146a in controlling Treg cell-mediated regulation of Th1 responses. Cell. 2010;142:914-29.

24. Volz HC, Laohachewin D, Schellberg D, Wienbrandt AR, Nelles $M$, Zugck $C$, et al. HMGB1 is an independent predictor of death and heart transplantation in heart failure. Clin Res Cardiol. 2012;101:427-35.

25. Pasupneti S, Khush K. Elevated troponin? Take heart and reconsider! Circulation: Heart Failure. 2016;9:e003237, https://doi.org/10.1161/CIRCHEARTFAILURE.116.003237.

26. Cen S, Wang P, Xie Z, Yang R, Li J, Liu Z, et al. Autophagy enhances mesenchymal stem cell-mediated CD4(+) T cell migration and differentiation through CXCL8 and TGF-beta1. Stem Cell Res Ther. 2019;10:265.

27. Huehn J, Beyer M. Epigenetic and transcriptional control of Foxp3+ regulatory T cells. Semin Immunol. 2015;27:10-8.

28. Rudra D, deRoos P, Chaudhry A, Niec RE, Arvey A, Samstein RM, et al. Transcription factor Foxp3 and its protein partners form a complex regulatory network. Nat. Immunol. 2012;13:1010-9.

29. Lan X, Wang G, Xu X, Lu S, Li X, Zhang B, et al. Stromal cell-derived factor-1 mediates cardiac allograft tolerance induced by human endometrial regenerative cell-based therapy. Stem Cells Transl Med. 2017;6:1997-2008.

30. Gardner D, Jeffery LE, Sansom DM. Understanding the CD28/CTLA-4 (CD152) pathway and its implications for costimulatory blockade. Am J Transplant. 2014;14:1985-91.

31. Srinivas TR, Kaplan B. Transplantation in 2011: new agents, new ideas and new hope. Nat Rev Nephrol. 2011;8:74-5.

32. Londono MC, Danger R, Giral M, Soulillou JP, Sanchez-Fueyo A, Brouard S. A need for biomarkers of operational tolerance in liver and kidney transplantation. Am J Transplant. 2012;12:1370-7.

33. Wood KJ, Bushell A, Hester J. Regulatory immune cells in transplantation. Nat Rev Immunol. 2012;12:417-30.

34. Hirschberger S, Hinske LC, Kreth S. MiRNAs: dynamic regulators of immune cell functions in inflammation and cancer. Cancer Lett. 2018;431:11-21.

35. Hall BM. Cells mediating allograft rejection. Transplantation 1991:51:1141-51.

36. Sakaguchi S, Yamaguchi T, Nomura T, Ono M. Regulatory T cells and immune tolerance. Cell 2008;133:775-87.
37. Bleriot C, Chakarov S, Ginhoux F. Determinants of resident tissue macrophage identity and function. Immunity 2020;52:957-70.

38. Zhang M, Nakamura K, Kageyama S, Lawal AO, Gong KW, Bhetraratana M, et al. Myeloid HO-1 modulates macrophage polarization and protects against ischemia-reperfusion injury. JCI Insight. 2018;3:e120596, https://doi.org/10.1172/ jci.insight.120596.

39. Tian J, Dang HN, Yong J, Chui WS, Dizon MP, Yaw CK, et al. Oral treatment with gamma-aminobutyric acid improves glucose tolerance and insulin sensitivity by inhibiting inflammation in high fat diet-fed mice. PLoS ONE. 2011;6:e25338.

40. Romano M, Fanelli G, Albany CJ, Giganti G, Lombardi G. Past, present, and future of regulatory $T$ cell therapy in transplantation and autoimmunity. Front Immunol. 2019;10:43.

41. Romano M, Fanelli G, Tan N, Nova-Lamperti E, McGregor R, Lechler Rl, et al Expanded regulatory $T$ cells induce alternatively activated monocytes with a reduced capacity to expand T helper-17 Cells. Front Immunol. 2018;9:1625

42. Tiemessen MM, Jagger AL, Evans HG, van Herwijnen MJ, John S, Taams LS. CD4 + CD25+Foxp3+ regulatory $T$ cells induce alternative activation of human monocytes/macrophages. Proc Natl Acad Sci USA. 2007;104:19446-51.

43. Gao C, Wang X, Lu J, Li Z, Jia H, Chen M, et al. Mesenchymal stem cells transfected with sFgl2 inhibit the acute rejection of heart transplantation in mice by regulating macrophage activation. Stem Cell Res Ther. 2020;11:241.

44. Goschl L, Scheinecker C, Bonelli M. Treg cells in autoimmunity: from identification to Treg-based therapies. Semin Immunopathol. 2019;41:301-14.

45. Zhang Q, Zhou X, Wan M, Zeng X, Luo J, Xu Y, et al. FoxP3-miR-150-5p/3p suppresses ovarian tumorigenesis via an IGF1R/IRS1 pathway feedback loop. Cell Death Dis. 2021;12:275.

46. Oh H, Grinberg-Bleyer Y, Liao W, Maloney D, Wang P, Wu Z, et al. An NF-kappaB transcription-factor-dependent lineage-specific transcriptional program promotes regulatory T cell identity and function. Immunity 2017;47:450-65 e5.

47. Long M, Park SG, Strickland I, Hayden MS, Ghosh S. Nuclear factor-kappaB modulates regulatory $\mathrm{T}$ cell development by directly regulating expression of Foxp3 transcription factor. Immunity 2009;31:921-31.

48. Liu R, Liu C, Chen D, Yang WH, Liu X, Liu CG, et al. FOXP3 controls an miR-146/NFkappaB negative feedback loop that inhibits apoptosis in breast cancer cells. Cancer Res. 2015:75:1703-13.

49. Liu R, Yi B, Wei S, Yang WH, Hart KM, Chauhan P, et al. FOXP3-miR-146-NF-kappaB axis and therapy for precancerous lesions in prostate. Cancer Res. 2015;75:1714-24

50. Zhou Q, Haupt S, Kreuzer JT, Hammitzsch A, Proft F, Neumann C, et al. Decreased expression of miR-146a and miR-155 contributes to an abnormal Treg phenotype in patients with rheumatoid arthritis. Ann Rheum Dis. 2015;74:1265-74.

51. Marinaro F, Marzi MJ, Hoffmann N, Amin H, Pelizzoli R, Niola F, et al. MicroRNAindependent functions of DGCR8 are essential for neocortical development and TBR1 expression. EMBO Rep. 2017;18:603-18.

52. Niimi M. The technique for heterotopic cardiac transplantation in mice: experience of 3000 operations by one surgeon. J Heart Lung Transplant. 2001;20:1123-8.

53. Stewart S, Fishbein MC, Snell GI, Berry GJ, Boehler A, Burke MM, et al. Revision of the 1996 working formulation for the standardization of nomenclature in the diagnosis of lung rejection. J Heart Lung Transplant. 2007;26:1229-42.

54. Mehra MR, Crespo-Leiro MG, Dipchand A, Ensminger SM, Hiemann NE, Kobashigawa JA, et al. International Society for Heart and Lung Transplantation working formulation of a standardized nomenclature for cardiac allograft vasculopathy-2010. J Heart Lung Transplant. 2010;29:717-27.

55. Li X, Lan X, Zhao Y, Wang G, Shi G, Li H, et al. SDF-1/CXCR4 axis enhances the immunomodulation of human endometrial regenerative cells in alleviating experimental colitis. Stem Cell Res Ther. 2019;10:204.

\section{ACKNOWLEDGEMENTS}

We are very grateful to Prof. Wenli Feng, Dr. Jie Gao, Dr. Shuwei Feng, and Dr. Xin Su for providing technical support. This work was supported by the National Natural Science Foundation of China (Grant No. 81570375), the "Thirteenth Five-Year Plan" Comprehensive Investment Discipline Construction Project (Grant No. 116015012016XK0301), and the Tianjin Binhai New Area Health Committee Science and Technology Innovation Support Project (Grant No. 2017BWKY036).

\section{AUTHOR CONTRIBUTIONS}

$\mathrm{L}$ designed and performed the majority of the experiments, and wrote the manuscript. WW and PL performed cytokine analyses. XW established the animal model. CG and BZ performed flow cytometry analyses. $X D$ and $Y L$ carried out pathological examinations. YY collected and analyzed the data. FQ designed the study and edited the manuscript. The final manuscript was read and approved by all authors. 
12

\section{COMPETING INTERESTS}

The authors declare no competing interests.

\section{ETHICS STATEMENT}

This study was approved by the Ethics Committee of Tianjin Medical University General Hospital.

\section{ADDITIONAL INFORMATION}

Supplementary information The online version contains supplementary material available at https://doi.org/10.1038/s41420-021-00534-9.

Correspondence and requests for materials should be addressed to F.Q.

Reprints and permission information is available at http://www.nature.com/ reprints
Publisher's note Springer Nature remains neutral with regard to jurisdictional claims in published maps and institutional affiliations.

(C) Open Access This article is licensed under a Creative Commons Attribution 4.0 International License, which permits use, sharing, adaptation, distribution and reproduction in any medium or format, as long as you give appropriate credit to the original author(s) and the source, provide a link to the Creative Commons license, and indicate if changes were made. The images or other third party material in this article are included in the article's Creative Commons license, unless indicated otherwise in a credit line to the material. If material is not included in the article's Creative Commons license and your intended use is not permitted by statutory regulation or exceeds the permitted use, you will need to obtain permission directly from the copyright holder. To view a copy of this license, visit http://creativecommons. org/licenses/by/4.0/.

(c) The Author(s) 2021 